\title{
Correlation of granularity index with toxic granulation of neutrophils by manual microscopy and $\mathrm{C}$-reactive protein
}

\author{
${ }^{1}$ Vaddatti Tejeswini, ${ }^{2}$ Sreenivasulu Kande, ${ }^{3}$ P. Premalatha, \\ ${ }^{4} \mathrm{~T}$. Rayapa reddy \\ .(Pathology,NRIMC\&GH, NTRUHS, India) ${ }^{[1]}$ (Pathology, NRIMC\&GH, NTRUHS, India) ${ }^{[2]}$ (Pathology, \\ NRIMC\&GH, NTRUHS, India $)^{[3]} .(\text { Pathology, NRIMC\&GH, NTRUHS, India })^{[4]}$
}

\begin{abstract}
Background:During inflammation there is increase in plasma concentrations of C Reactive Protein (CRP) along with appearance of toxic granulation neutrophils(TGNs) in the peripheral blood. The granularity of TGNs are graded according to their intensity by manual microscopy. Granularity index(GI) of neutrophils calculated by SEIMENS ADVIA 2120 measures the intensity of neutrophilic granules.

Aim: The objective of the present study is to evaluate if GI-Index correlates with TGNs and plasma CRP levels.

Materials And Methods: In 228 patients TGNs, GI-Index and CRP were determined. TGNs was graded in Giemsa stained peripheral blood smears, by manual microscopy using a newly proposed grading system. GIIndex calculated by SEIMENS ADVIA 2120, as a quantitative measure from NEUTX values. CRP levels estimated by turbidometry.

Results: The samples were analysed and compared with Pearson's coefficient of correlation( $r)$. There was statistically significant correlation between GI index and grading of TGNs $(n=228 ; r=0.723 ; p<0.0001)$. The correlation of GI index and CRP was positive but less significant ( $n=228 ; r=0.371 ; p<0.0001$ ) probably due to variation in the extent and cause of inflammation.

Conclusions: We conclude from this study that GI Index can be used to replace labour intensive, less reliable and hardly reproducible grading of TGNs by manual microscopy, as an indicator of infection. Additional inflammatory markers like plasma CRP levels are used as adjuvant to GI Index.
\end{abstract}

Key words: CRP, GI-Index, NEUTX, TGNs.

\section{Introduction}

Infection often leads to increased prominence of azurophilic granules of neutrophils.[1] and plasma $\mathrm{C}$ Reactive Protein(CRP). NEUT X derived from SEIMENS ADVIA 2120 is representative of the structure and granularity of the neutrophils, [2] based on this the Granularity Index (GI-Index) was defined. ${ }^{[2,3]}$ According to the intensity of granules, toxic granulation of neutrophils (TGNs) were graded. Though correlation of TGNs with CRP is recognised in infections, only limited data is available regarding GI-Index and TGNs. Hence the present study was done to evaluate GI-Index, a simple, highly reliable and reproducible marker to replace TGNs and plasma CRP.

II. Aim

The aim of this study was to evaluate the GI-Index as a parameter for toxic granulation in acute inflammation or infection to replace the labor intensive, highly subjective and only semiquantitative manual microscopic procedure. The relationship between granularity of TGNs in peripheral blood smears, the GI-Index and plasma $\mathrm{C}$-reactive protein (CRP) concentrations was examined

\section{Materials And Methods}

The present study was conducted between February 2011 to April 2011 in a tertiary care teaching hospital (NRIMC\&GH, Chinakakani) with 1000 beds. In this study 228 blood samples from patients suspected to have infections or any inflammatory conditions were analysed for complete blood picture by SEIMENS ADVIA 2120. Peripheral blood smears prepared and stained with Wright Giemsa stain to grade intensity of TGNs. The samples were also subjected to evaluate plasma CRP levels by turbidometry.

Morphological examination of neutrophils was done under magnifications of 400x and 1000x. Minimum of 50 neutrophils counted per slide and an average impression was obtained and categorized. Neutrophils with toxic granules were defined by the presence of dark blue to purple coloured granules in the cyptoplasm. Toxic granules were graded into 5 categories $(0,1+, 2+, 3+, 4+$.$) depending on the staining$ intensity proposed by A van de Vyver, E F Delport, M Esterhuizen, $R$ Pool as in table 1. [4] 
NEUT X values from all the samples tabulated to calculate granularity index (GI) of neutrophils. The mean value of Side Scatter measurement of the neutrophil population (NEUTX) which is representative of the structure of the neutrophils, could be used to calculate Granularity index (GI) . After analyzing 50 healthy reference population, the resulting mean NEUT-X ( Mean 49.6, SD-2.34) was defined to equate to a GI Index value of 0 . NEUT-X +/- 1 SD corresponds to a GI of zero, NEUT-X lower than 1 SD from the mean would give a GI of -1 and NEUT-X higher than 1 SD from the mean would give a GI of +1 . A GI-Index of one represents hypergranulation with a NEUT-X value of one standard deviation above the mean. A GI-Index of -1 reflects hypogranulation with a NEUT-X value of one standard deviation below the mean NEUT-X of the reference population, respectively. ${ }^{[2,3]}$ GI scoring system is depicted in table 2. ${ }^{[5]}$ This study investigated if it was possible to use the quantitative expression of NEUT-X as a alternative for microscopic grading of TGNs in the routine laboratory.

The plasma C Reactive Proteins were assessed by turbidometric method. The value of $>6$ was considered positive

All the 3 parameters were evaluated to assess possible trend or correlation.

\section{[3.1] Inclusion Criteria:}

Samples were analysed from patients with suspected or confirmed infection and where the clinician has requested for full blood count, differential count and plasma CRP levels.

\section{[3.2] Exclusion Criteria:}

Suspected or confirmed cases of leukaemias were excluded from the study.

\section{Statistics}

228 samples were analysed for complete blood picture, GI-Index, TGNs and CRP. The statistical analysis was done by SSPE and expressed as Mean, SD, Pearson's coefficient of correlation ( $r$ ) between the parameters. $r$ of $0-1$ is considered as positive correlation with $>0.5$ as strong correlation. $r<0$ indicates negative correlation. 'p' value calculated, $<0.0001$ was considered statistically significant.

\section{Results}

In total 228 blood samples were analysed in the present study for full blood counts, differential count, grading of toxic granulation of neutrophils (TGN), granularity index (GI) and serum CRP levels. Samples of varying ages, minimum age of 1 day and maximum age of 80 years were included. Maximum number of cases was seen in $6^{\text {th }}$ decade consisting of $24.12 \%$. Male preponderance was seen with male to female ratio being 1.37: 1 . The age and sex distribution is depicted in table 3 .

GI Index was compared with TGNs and CRP. GI Index ranged from -1 to +4 . The grading of TGNs was performed as in table $1(0,1$, and 2). The characteristics of TGN grading \& CRP levels in various groups of GI Index is shown in table 4. Most of the samples included were of GI Index 2 (88 cases, consisting of 38.59\% of total cases). We observed that GI Index rises with an increasing grade of TGNs granularity in the blood smear. The GI-Index displayed a significant correlation $(\mathrm{n}-228 ; \mathrm{r}-0.72 ; \mathrm{p}<0.0001)$ with the granularity of TGNs. The two parameters were evaluated using Pearson's coefficient of correlation(r) and 'p' value calculated. The plasma CRP concentrations ranged from 0.04- $69.68 \mathrm{IU} / \mathrm{ml}$. The value of > $6 \mathrm{IU} / \mathrm{ml}$ was considered significant. A correlation analysis of the GI Index with the CRP concentration revealed a positive correlation (n228; r-0.37; t-5.99; $\mathrm{p}<0.0001)$. Though the Pearson's coefficient of correlation of GI Index and CRP ( $\mathrm{r}-0.37)$ is positive but less significant statistically. This can be explained as plasma CRP levels are not solely diagnostic of infection. The values are also increased in other causes of inflammation and tissue damage due to release of cytokines. The statistical correlation between GI Index, TGNs and plasma CRP values is depicted in table 5 .

\section{Discussion}

The relationship between toxic granules in mature neutrophils and high CRP levels in infectious disease and inflammation has been described. [6] However, the relationship between the GI Index of neutrophils, intensity of TGNs and CRP levels has not been studied extensively. In our present study, GI score along with the graded intensity of TGNs in neutrophils was evaluated using a newly designed scale which was then compared with the plasma CRP values in patients with possible inflammatory or infectious disease.

Acute infections and inflammatory conditions usually show elevated counts of leukocytes and neutrophils. The production of neutrophils is stimulated by G-CSF, GM-CSF, IL-3 and M-CSF. [7] The granules are divided into primary which appear at promyelocyte stage and secondary which appear at myelocyte stage and predominate in mature neutrophil. ${ }^{[8]}$ Tertiary granules include secretory vescicles with alkaline phosphatase and gelatinase granules. The use of neutrophil quantitative parameters, and qualitative changes as indicators for infective disease has been evaluated previously, but has limited value. ${ }^{[9]}$ The role of leukocytosis 
and neutrophilia in predicting bloodstream infection is also of questionable utility, as their etiologies include infection, stress, medication, trauma, and abnormal bone marrow production. ${ }^{[10]}$ These neutrophils show toxic granules with shift to left. Kugel and Rosenthal described in 1932 the presence of "large, dark, irregular, basophilic granules" in the cytoplasm of neutrophilic granulocytes in patients with severe infection in comparison to the typical fine granular staining pattern. These were named as "toxic granules" (TG) and are helpful in predicting acute infection. Determining the intensity of TG is laborious and the reproducibility is potentially poor, owing to inter- and intra-observer variability. Grading TG is dependent on technique, training and the experience of the haematologist. It has been suggested that the total white blood cell count and absolute neutrophil count should be monitored because these parameters yield more reproducible results. Despite these difficulties, most laboratories still use morphological changes in neutrophils (i.e. TG and vacuolisation) on peripheral blood smears as a diagnostic tool, especially to predict acute pyogenic infections. Liu et al. described the usefulness of these morphological changes, that the presence of TG was more predictive of bacteraemia than the total leukocyte count. ${ }^{[9]}$ Sabah et al. found toxic changes in neutrophils to be highly sensitive, which was confirmed by Al-Gwaiz and Babay, who found a sensitivity of $78 \%$ for detecting acute bacterial infection. In contrast, toxic granules of granulocytes were absent in blood smears of patients with mild or localized infections. Toxic granulation neutrophils (TGNs) are a well-known but not adequately understood phenomenon. TGNs become apparent during inflammatory processes, in blood smears stained by the May- Gru"nwaldGiemsa (MGG) or Wright technique in manual microscopy.

SEIMENS ADVIA 2120, an automated analyser assesses neutrophil activation in EDTA anticoagulated blood samples based on cell granularity, lipid membrane components, cell shape, volume and total nucleic acid compounds. ${ }^{[11]}$ Different fluorescent dyes (polymethines and oxazine dyes) stain nucleic acids as well as cellular protein components of blood cells. Membrane surfactant reagents perforate cell membranes yielding information about cell-shape and generation of bioactive lipids in cell membranes. Sideward and forward-scatter measurements indicate cellular granularity and size of blood cells. ${ }^{[11]}$ Using fluorescence flow cytometry techniques, by SEIMENS ADVIA 2120 neutrophil activation can be measured as hypo- or hypergranulation by change of polymorphonuclear neutrophilic granulocytes (PMN) granularity (NEUT-X in the DIFF channel, which is the direct measurement of side scatter diffraction.). ${ }^{[2,11,12]}$ GI is calculated from NEUT X value. A low NEUT-X and GI strongly correlates with hypogranularity in the neutrophils and vice versa. NEUT-X was not found to correlate to the neutrophil count, even in quite severe neutropenia. ${ }^{[13]}$ As GI is a measure of granularity of neutrophils, it has been compared with grades of TGNs.. Calculating GI is less labourious, more accurate, highly reproducible and reliable when compared to TGNs. Hence in this study we tried to evaluate if GI can be used as an alternative parameter for microscopic grading of TGNs.

As clinical symptoms and conventional markers are not always reliable signs for the diagnosis of sepsis and infection, biomarkers such as CRP or procalcitonin (PCT) are often used as a diagnostic tool in these patients. CRP was the first acute-phase protein to be described and is an exquisitely sensitive systemic marker of inflammation and tissue damage. ${ }^{[14]} \mathrm{CRP}$ levels can be markedly elevated with tissue necrosis that is not necessarily associated with infection. Conversely, normal to moderate elevation can be seen in patients with infection and underlying liver disease and certain auto-immune diseases such as systemic lupus erythrematosus, scleroderma and ulcerative colitis. ${ }^{[14]}$ PCT levels increase far less than CRP levels and the period of unspecific induction is much shorter. The PCT parameter is therefore the better choice to diagnose sepsis and infection early after surgery. ${ }^{[15,16]}$ The total leukocyte count cannot be used as a marker for infection because certain drugs like corticosteroids, produces falsely elevated counts, by preventing migration to the tissue pool. [7] Therefore, a tool is needed that can be used to monitor infection in conditions affecting liver and renal function, associated with underlying inflammatory processes, with possible concurrent corticosteroid therapy. The degree of TG can be a useful adjunct in this setting, where most other markers have been confounded. As TGNs is subjective and variable manual microscopic procedure, we tried to replace this with GI Index in this study. We found a statistically significant trend between GI-Index, grading the intensity of TGNs and a rise in CRP. Statistically significant differences in GI score were found between the graded TGNs categories. GI Index is well correlated with morphological changes in neutrophils like TGNs and therefore can be used as a suitable parameter in evaluating disease severity in patients with underlying inflammatory or infectious conditions. A less significant positive correlation was found between GI Index and CRP. Hence along with GI Index , CRP can be used as additional inflammatory marker. 


\section{Conclusions}

The above results confirm a trend in the rise of GI index, as the intensity of TGNs in peripheral blood increases along with rise in serum levels of CRP. The GI-Index is suited to quantify the granularity of TGNs. The GI-Index is an automated, standardized, highly reliable and accurate parameter. We suggest that it replace the time-consuming, subjective and semiquantitative microscopic procedure. As CRP is not diagnostic on its own , this can be used only as additional marker.

\section{Tables}

Table 1 Grading of toxic granulation neutrophils (TGNs)[4]

\begin{tabular}{|l|l|}
\hline Grade & Morphology \\
\hline 0 & Normal granulated neutrophils \\
\hline 1 & Scattered granules in the cytoplasm with associated increase in stain intensity \\
\hline 2 & Increased number of granules in the cytoplasm with associated increase in stain intensity \\
\hline 3 & Numerous granules in the cytoplasm with intense blue black staining properties \\
\hline 4 & Numerous coarse granules crowding the cytoplasm \\
\hline
\end{tabular}

After initial analysis into 5 categories, they were combined and grouped as 0 ( no toxic granules), 1 (grade $1 \&$ 2), 2 ( grade $2 \& 3$ ) for stastistical purpose.

Table 2 The calculation of the granularity index based on deviations from the mean value for NEUT-X found in the normal control groups of patients

\begin{tabular}{|l|l|l|l|l|l|l|l|l|l|l|}
\hline $\begin{array}{l}\text { Difference } \\
\text { of NEUT-X } \\
\text { from } \\
\text { reference } \\
\text { value }\end{array}$ & $\begin{array}{l}37.90- \\
40.24\end{array}$ & $\begin{array}{l}40.24- \\
42.58\end{array}$ & $\begin{array}{l}42.58-92 \\
44.92\end{array}$ & $\begin{array}{l}44.92- \\
47.26\end{array}$ & $\begin{array}{l}47.26- \\
51.94\end{array}$ & $\begin{array}{l}51.94- \\
54.28\end{array}$ & $\begin{array}{l}54.28- \\
56.62\end{array}$ & $\begin{array}{l}56.62- \\
58.96\end{array}$ & $\begin{array}{l}58.96- \\
61.3\end{array}$ \\
\hline GI Index & -4 & -3 & -2 & -1 & 0 & +1 & +2 & +3 & +4 \\
\hline
\end{tabular}

Table 3 - Age / Sex distribution

\begin{tabular}{|l|l|l|l|}
\hline Age/ Sex & Male & Female & Total \\
\hline $0-10$ & 36 & 16 & 52 \\
\hline $11-20$ & 03 & - & 03 \\
\hline $21-30$ & 07 & 08 & 15 \\
\hline $31-40$ & 13 & 12 & 25 \\
\hline $41-50$ & 31 & 21 & 52 \\
\hline $51-60$ & 27 & 28 & 55 \\
\hline $61-70$ & 11 & 07 & 18 \\
\hline $71-80$ & 04 & 04 & 08 \\
\hline Total & 132 & 96 & 228 \\
& & & \\
\hline
\end{tabular}

Table 4 _TGN grading \& CRP charactertistics in GI Index

\begin{tabular}{|l|l|l|l|l|l|l|l|}
\hline $\begin{array}{l}\text { GI Index/ } \\
\text { Group }\end{array}$ & $\begin{array}{l}\text { No of of } \\
\text { samples }\end{array}$ & $\begin{array}{l}\text { TGN } \\
\text { Mean }\end{array}$ & $\begin{array}{l}\text { CRP } \\
\text { Mean }\end{array}$ & $\begin{array}{l}\text { Minimum } \\
\text { TGN }\end{array}$ & $\begin{array}{l}\text { Minimum } \\
\text { CRP }\end{array}$ & $\begin{array}{l}\text { Maximum } \\
\text { TGN }\end{array}$ & $\begin{array}{l}\text { Maximum } \\
\text { CRP }\end{array}$ \\
\hline-1 & 1 & 0 & 0.6 & 0 & 0.6 & 0 & 0.6 \\
\hline 0 & 32 & 0.25 & 2.542 & 0 & 0.04 & 1 & 19.45 \\
\hline 1 & 84 & 0.726 & 3.067 & 0 & 0.1 & 3 & 33.42 \\
\hline 2 & 88 & 1.477 & 8.551 & 0 & 0.08 & 3 & 68.17 \\
\hline 3 & 22 & 2.545 & 14.458 & 1 & 2.06 & 3 & 69.68 \\
\hline 4 & 1 & 3 & 68.5 & 3 & 68.5 & 3 & 68.5 \\
\hline
\end{tabular}

Table 5- Correlation of GI Index with TGNs and plasma CRP

\begin{tabular}{|l|l|l|}
\hline Group & Pearson's correlation of coefficient $(\mathrm{r})$ & 'p'value \\
\hline TGNs & 0.72 & $<0.0001$ (significant) \\
\hline CRP & 0.37 & $<0.0001$ (significant) \\
\hline
\end{tabular}




\section{Acknowledgements}

We are greatly thankful to Dr.R. Krishna, Dr. PAV Krishnamacharyulu, Dr. S. Satyanarayana Rao, Professors of pathology, Dr. N. Swathantra, Asst. professor of pathology for giving their valuable suggestions for this work and Dr.K. Anji Reddy, Dean of NRIMC\&GH, Dr. NVS Choudary, Principal of NRIMC for allowing and providing required material for the research

\section{References}

[1] John P.Greer, John Foerster, John N. Lukens, George M. Rodger, Frixos Paraskevas, Bertil Glader. Wintrobes clinical haematology, $11^{\text {th }}$ edition, Lippincott Williams \& Wilkins. 2004, 15

[2] Le Roux G, Vlad A, Eclache V, Malanquin C, Collon JF, Gantier M, et al. Routine diagnostic procedures of myelodysplastic syndromes: value of a structural blood cell parameter (NEUT-X) determined by the Sysmex XE-2100TM. Int J Lab Hematol2010;32:e237-43.

[3] Furundarena JR, Araiz M, Uranga M, Sainz MR, Agirre A, Trassorras M, et al. The utility of the Sysmex XE-2100 analyzer's NEUT-X and NEUT-Y parameters for detecting neutrophil dysplasia in myelodysplastic syndromes. Int $\mathrm{J}$ Lab Hematol 2010;32:360-6.

[4] A van de Vyver, E F Delport, M Esterhuizen, R Pool.The correlation between C-reactive protein and toxic granulation of neutrophils in the peripheral blood. SAMJ July 2010, Vol. 100, No. 7. 442-444

[5] F. Cymbalista. Usefulness of neut-x determination in routine diagnostic procedures: application to myelodysplasticsyndromes. Diagnostic Perspectives - The Sysmex Europe e Journal for Clinical Laboratory Medicine · Published 2010 by Sysmex

[6] Kim YS, Park HH, Rhee HW, et al. Neutrophils with toxic granulation shows high

[7] fluorescence with Bis (ZN2+-dipicolylamine) complex. Ann Clin Lab Sci 2009; 39(2): 114-119.

[8] Hoffbrand AV, Catovsky D, Tuddenham EGD. Postgraduate Haematology. 5th ed. Oxford, UK: Blackwell Publishing, $2005: 286$.

[9] A.V.Hoffbrand, P.A.H. Moss and J.E.Pettit. Essential haematology. $5^{\text {th }}$ edition.

[10] Liu CH, Lehan C, Speer ME. Early detection of bacteremia in an outpatient clinic. Pediatrics 1985; 75: 827-831.

[11] Clinton K. Murray, MD; Roselle M. Hoffmaster, MD; David R. Schmit, BS; Duane R. Hospenthal, MD, PhD; John A. Ward, PhD; Leopoldo C. Cancio, MD; Steven E.Wolf, MD. Evaluation of White Blood Cell Count, Neutrophil Percentage, and Elevated Temperature as Predictors of Bloodstream Infection in Burn Patients. Arch Surg. 2007;142(7):639-642.

[12] Linssen J, Aderhold S, Nierhaus A, Frings D, Kaltschmidt C, Zänker K. Automation and validation of a rapid method to assess neutrophil and monocyte activation by routine fluorescence flow cytometry in vitro. Cytometry Part B 2008;74B:295-309.

[13] Mathias Zimmermann, Malte Cremer, Christina Hoffmann, Karin Weimann and Andreas Weimann. Granularity Index of the SYSMEX XE-5000 hematology analyzer as a replacement for manual microscopy of toxic granulation neutrophils in patients with inflammatory diseases. Clin Chem Lab Med 2011;49(7):1193-1198 _ 2011

[14] Diagnostic Perspectives - The Sysmex Europe e Journal for Clinical Laboratory Medicine. Published 2010 by Sysmex

[15] Pepys MB, Hirschfield M. C-reactive protein: a critical update. J Clin Invest 2003; 111: 1805-1812.

[16] Michael Meisner, Heide Adina and Joachim Schmidt. Correlation of procalcitonin and C-reactive protein to inflammation, complications, and outcome during the intensive care unit course of multiple-trauma patients. Critical Care Vol 10 No 1 